\title{
The Role of Filler-Matrix Interaction on Viscoelastic Response of Biomimetic Nanocomposite Hydrogels
}

\author{
Alireza S. Sarvestani, Xuezhong He, and Esmaiel Jabbari \\ Biomimetic Materials and Tissue Engineering Laboratories, Department of Chemical Engineering, \\ University of South Carolina, Columbia, SC 29208, USA \\ Correspondence should be addressed to Esmaiel Jabbari, jabbari@engr.sc.edu
}

Received 19 September 2007; Accepted 2 May 2008

Recommended by Hongchen Gu

The effect of a glutamic acid (negatively charged) peptide (Glu6), which mimics the terminal region of the osteonectin glycoprotein of bone on the shear modulus of a synthetic hydorgel/apatite nanocomposite, was investigated. One end of the synthesized peptide was functionalized with an acrylate group (Ac-Glu6) to covalently attach the peptide to the hydrogel phase of the composite matrix. The addition of Ac-Glu6 to hydroxyapatite (HA) nanoparticles (50 $\mathrm{nm}$ in size) resulted in significant reinforcement of the shear modulus of the nanocomposite ( $\sim 100 \%$ increase in elastic shear modulus). The reinforcement effect of the Glu6 peptide, a sequence in the terminal region of osteonectin, was modulated by the size of the apatite crystals. A molecular model is also proposed to demonstrate the role of polymer-apatite interaction in improving the viscoelastic behavior of the bone mimetic composite. The predictions of the model were compared with the measured dynamic shear modulus of the PLEOF hydrogel reinforced with HA nanoparticles. This predictive model provides a quantitative framework to optimize the properties of reinforced polymer nanocomposites as scaffolds for applications in tissue regeneration.

Copyright ( $) 2008$ Alireza S. Sarvestani et al. This is an open access article distributed under the Creative Commons Attribution License, which permits unrestricted use, distribution, and reproduction in any medium, provided the original work is properly cited.

\section{INTRODUCTION}

Synthetic degradable and biomimetic polymer nanocomposites are an ideal replacement material for orthopedics and dental applications because of minimum risk of disease transfer, reduced stress shielding and particulate wear, and the ability to couple polymer degradation with tissue regeneration. In particular, injectable hydrogels seeded with cells and growth factors and coupled with minimally invasive arthroscopic techniques are an attractive alternative for treating irregularly shaped degenerated hard tissues. Marrow stromal cells, isolated from the bone marrow, and growth factors can be placed in a supportive hydrogel and injected into an osteochondral defect by a minimally invasive arthroscopic procedure [1-5].

After injection, the composite mixture hardens in situ, guiding the development of the seeded cells into the desired tissue. Furthermore, the composite matrix provides dimensional stability and mechanical strength, similar to that of the host tissue, during regeneration. A variety of multifunctional composite materials have been developed to mimic the organized nanostructure of the bone, which consists of the collagenous matrix and mineralized apatite nanocrystals [6-8]. In addition, the gelatinous bone matrix contains noncollagenous proteins (NCPs), which play a central role in regulation of mineralization and the extent of mineral-collagen interactions $[9,10]$. One of the NCPs with bone specific functions is osteonectin which has a strong affinity for both collagen and hydroxyapatite (HA), and it is speculated to be a bone-specific nucleator of mineralization $[11,12]$. It is believed that the first seventeen $\mathrm{NH}_{2}$-terminal amino acids of osteonectin are responsible for binding to the bone collagen network [13], while a glutamic acid-rich sequence binds to the bone HA nanoparticles, due to its high ionic affinity for calcium ions [12].

In this work, we describe the synthesis and rheological characterization of a multifunctional bone mimetic nanocomposite with a matrix-apatite adhesion mechanism similar to that of the natural bone. We have synthesized a glutamic acid-rich peptide (a sequence of 6 glutamic acids) 


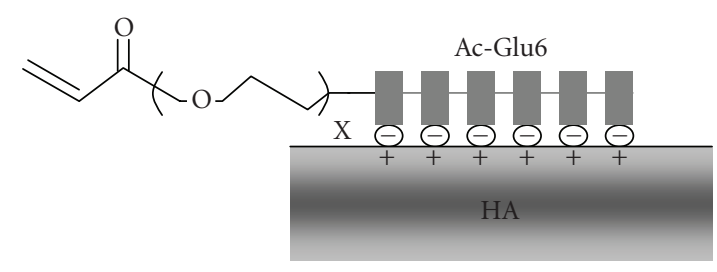

Figure 1: Schematic structure of the Ac-Glu6 peptide used for surface treating of HA nanoparticle. Terminal acrylate group of the Ac-Glu6 provides an unsaturated group for covalent cross-linking of the apatite particles to the PLEOF matrix.

derived from osteonectin, functionalized with an acrylate group for covalent attachment to the matrix, using solidphase Fmoc chemistry [14]. The biodegradable in situ crosslinkable poly (lactide-co-ethylene oxide fumarate) (PLEOF) hydrogel and HA crystals were used to mimic the gelatinous matrix and mineral phases of the bone, respectively. PLEOF is a degradable macromer consisting of ultra-lowmolecular-weight poly (L-lactide) (ULMW PLA) and poly (ethylene glycol) (PEG) blocks li nked by fumaric acid. HA particles were treated with the synthesized glutamic acid peptide with acrylate group at one chain-end (Figure 1), hereafter designated as Ac-Glu6, and the nanoparticles were dispersed in the aqueous PLEOF mixture by sonication. The polymerizing mixture was cross-linked with a neutral redox initiation system and the gelation process was monitored by monitoring the viscoelastic response in situ as a function of gelation time with a rheometer. The measured rheological and viscoelastic characteristics can be used to control the injectability in the in situ hardening phase and to predict mechanical properties in the postgelation phase.

The rheometry results show that the Ac-Glu6 peptide can significantly enhance the viscoelastic properties of the hydrogel/HA nanocomposite. A molecular model is also proposed to demonstrate the role of polymer-apatite interaction in improving the viscoelastic behavior of the synthesized bone mimetic hydrogel. The scaling law of de Gennes for equilibrium reversible polymer adsorption in good solvent conditions [15] is used to predict the equilibrium configuration of the adsorbed polymer layer on the surface of HA particles. The relaxation and diffusion of the adsorbed segments, and consequently their flow characteristics, are predicted using a Maxwell type kinetic model. Predictions of the model are compared with the measured dynamic shear modulus of the PLEOF hydrogel reinforced with HA nanoparticles. This predictive model can provide a quantitative framework to design and optimize the properties of reinforced polymer composites as scaffolds for applications in tissue regeneration.

\section{EXPERIMENTAL METHODS}

\subsection{Synthesis of PLEOF}

PLEOF was synthesized by condensation polymerization of ULMW PLA [16] and PEG with fumaryl chloride ( $\mathrm{FuCl})$, as shown in Figure 2. The procedure for synthesis is described in a previous publication [16]. The weight ratio of PLA to PEG was 30/70 to produce a hydrophilic PLEOF macromer. The structure of the macromer was characterized by ${ }^{1} \mathrm{H}$ NMR and GPC. The synthesized PLEOF had $M_{n}$ and PI values of $10.5 \mathrm{kDa}$ and 1.7 , respectively, as determined by GPC.

\subsection{Synthesis of Ac-Glu6 peptide}

The functionalized Ac-Glu6 peptide, a negatively charged Glu-Glu-Glu-Glu-Glu-Glu peptide sequence with an acrylate group at one chain-end (Figure 1), was synthesized manually in the solid phase on the Rink Amide NovaGel resin [14]. Briefly, the Fmoc-protected amino acids were coupled to the resin in $\mathrm{N}, \mathrm{N}$-dimethylformamide using $\mathrm{N}, \mathrm{N}^{\bullet}$ diisopropylcarbodiimide and $N, N$-dimethylaminopyridine as the coupling agents. After coupling the last amino acid, the Fmoc-protecting group of the last glutamine residue was selectively deprotected with piperidine. One end was acrylated directly on the peptidyl resin by coupling acrylic acid to the amine group of the last glutamine residue in the peptide sequence. The resin was treated with $95 \%$ TFA/2.5\% TIPS/2.5\% water for 2 hours to cleave the peptide from the resin. The solution was precipitated in ether, the solid was purified by preparative HPLC (Waters, Milford, Mass, USA), and the product was freeze-dried. The product was characterized by mass spectrometry with a Finnigan 4500 spectrometer [14]. A similar procedure was used to synthesize the neutral Gly-Gly-Gly-Gly-Gly-Gly (Gly6) peptide and positively-charged Lys-Lys-Lys-Lys-LysLys (Lys6) peptide sequences with an acrylate group at one chain-end (Ac-Gly6 and Ac-Lys6, resp.).

\subsection{Preparation of the hydrogel nanocomposite}

$100 \mathrm{mg}$ Ac-Glu6 peptide $\left(M_{n}=900 \mathrm{Da}\right)$ was dissolved in $0.825 \mathrm{~mL}$ of distilled deionized (DDI) water by vortexing and heating the mixture to $50^{\circ} \mathrm{C}$. HA filler (Berkeley Advanced Biomaterials, Berkeley, Calif, USA) with average size of $50 \mathrm{~nm}$ (measured by TEM), with volume fraction ranging from 3 to $9 \%\left(\rho_{\mathrm{HA}}=3.16 \mathrm{~g} / \mathrm{cm}^{3}\right)$ was added to the PLEOF polymerizing mixture and the resulting dispersion was sonicated for 5 minutes. Larger spherical particles, with average diameter of $5 \mu \mathrm{m}$, were also used to investigate the effect of particle size. The composite mixture was prepared by dispersing the HA/Ac-Glu6 in PLEOF macromer $(0.04 \mathrm{M})$ and methylene bisacrylamide crosslinker $(0.25 \mathrm{M})$. The neutral redox initiation system with equimolar concentrations $(0.03 \mathrm{M})$ of ammonium persulfate and tetramethylethylenediamine was used to maintain the $\mathrm{pH}$ of the polymerizing mixture constant at 7.4.

\subsection{Rheological measurements}

The composite mixture was injected on the Peltier plate of the rheometer for rheological and gelation measurements. The dynamic storage modulus $\left(G^{\prime}\right)$ was measured at $37^{\circ} \mathrm{C}$ by a TA instrument AR2000 rheometer equipped with a parallel plate geometry $($ diameter $=20 \mathrm{~mm})$. A sinusoidal 


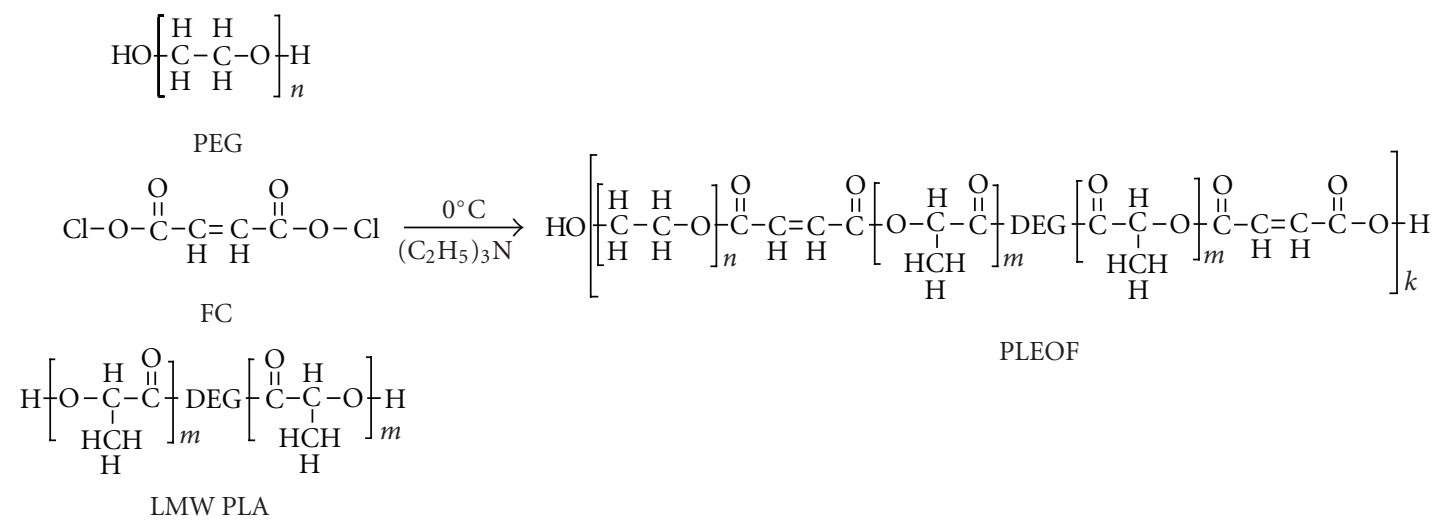

FIGURE 2: Schematic structure of the PLEOF macromer. DEG in the structure of PLEOF is diethylene glycol used as initiator in the synthesis of ULMW PLA by ring-opening polymerization of L-lactide monomer.

shear strain profile was exerted on the sample via the upper plate. The time sweep oscillatory shear measurements were done at constant frequency of $1 \mathrm{~Hz}$ and deformation amplitude equal to $1 \%$ for 3 hours. Each measurement was immediately followed by an amplitude sweep in the range increasing from $0.1 \%$ to $10 \%$ strain at frequency of $1 \mathrm{~Hz}$. To reduce the effect of particle aggregates disruption on the high strain nonlinear response of the composite, each amplitude sweep measurement was repeated with 30 minutes relaxation between the two runs and the results of the second run are reported here. Measurement for the third time revealed no difference between the last two measurements.

\section{EXPERIMENTAL RESULTS}

Figure 3 shows the variation of the normalized storage modulus of the hydrogel composites (recorded at the end of time sweep measurements) as a function of particle concentration for different particle size and surface treatment. Nanoapatite composites (treated and untreated) displayed far larger stiffness compared with microcomposites, at the same volume fraction. Shear modulus of the nanocomposites with Ac-Glu6 linker was higher than those without the apatite linker. The modulus of the composites with micron size particles did not appreciably change with the addition of Ac-Glu6. The contribution of hydrodynamic effect to the modulus of the composites can be predicted by GuthSmallwood equation [17], $G_{0}^{\prime}(\Phi)=G_{0}^{\prime}(0)(1+2.5 \Phi)$, where $G_{0}^{\prime}(0)$ and $G_{0}^{\prime}(\Phi)$ are the storage modulus of the gel and composite, respectively, and $\Phi$ is the filler volume fraction. The storage modulus of the composites prepared with micron size particles can be reasonably predicted by GuthSmallwood equation, as shown in Figure 3. However, the large difference between the experimental results and prediction of Guth-Smallwood equation for composites prepared with nanosize HA implies that the reinforcement cannot be explained solely by hydrodynamic effects in nanoparticulate systems.

To provide further evidence for energetic affinity between the Ac-Glu6 peptide and HA and its effect on the shear modulus of the nanocomposites, similar measurements were

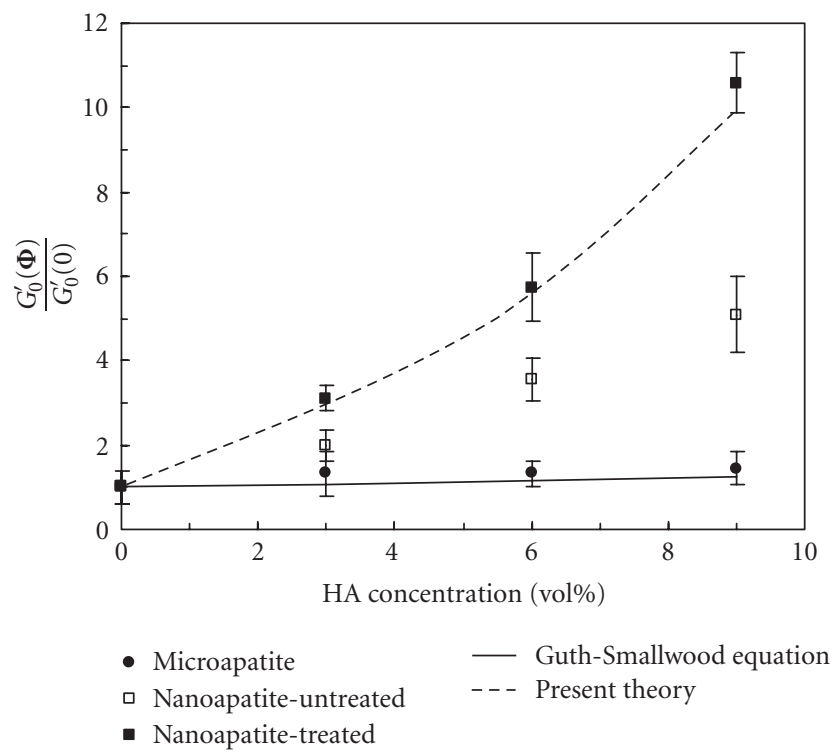

FIGURE 3: Dependence of the shear modulus of PLEOF/HA hydrogel composites on the size of the dispersed apatite particles. The normalized low-amplitude shear modulus predicted by the presented theory (dashed line) and Guth-Smallwood equation (solid line) are also compared with experimental data.

performed on the PLEOF/HA composites treated with equal molar concentrations of Ac-Gly6 and Ac-Lys6 in place of Ac-Glu6. Contrary to Glu6 peptide, Gly6 and Lys6 are neutral and positively charged, respectively. HA/Ac-Gly6 and HA/Ac-Lys6 nanocomposites with $9 \mathrm{vol} \%$ apatite did not show a significant change in storage shear modulus compared to that without HA surface treatment, as shown in Figure 4. The Ac-Lys6 is a positively charged sequence and it is expected to interact with the phosphate groups on the apatite surface in the same way that the negatively charged Ac-Glu6 sequence interacts with calcium ions, but the results in Figure 4 do not support this expectation. The charge ratio of $\mathrm{ca}^{2+}$ to $\mathrm{PO}_{4}{ }^{3-}$ groups in the $\mathrm{HA}$ crystal, with atomic composition $\left[\mathrm{Ca}_{10}\left(\mathrm{PO}_{4}\right)_{6}(\mathrm{OH})_{2}\right]$, is greater than one and 
to meet the requirement for electroneutrality, the negatively charged $\mathrm{OH}^{-}$groups compensate for the imbalance [18]. Electronic structure and interatomic potential-based calculations show that the $\mathrm{OH}^{-}$groups, in the bulk as well as the HA surface, are easily replaced by negatively charged fluoride ions [18]. Furthermore, phosphate and fluoride ions have been demonstrated to alter the mineral-organic interactions and influence the mechanical properties of the bone [19]. It is well established that certain small anionic molecules and polymers like poly (vinyl phosphonic acid) [20] and poly (acrylamide-co-acrylic acid) [21] displace negativelycharged hydroxyl groups (and in some cases phosphate groups) to interact and bond with calcium ions on the apatite surface. Based on these previous results, we believe that the Ac-Glu6 sequence ionically interacts with the apatite crystals by replacing the weakly bound hydroxyl groups (and perhaps the surface bound phosphate groups) from the surface but the same mode of interaction is not energetically favorable for Ac-Lys6, that is, Ac-Lys6 cannot replace the positively-charged surface calcium ions to interact with the apatite crystals. These results demonstrate that the increase in adsorption energy and its effect on the overall viscoelastic response of the nanocomposite are specific to the AcGlu6 peptide. The reinforcement is amplified as the size of the nanoparticles is reduced from $5 \mu \mathrm{m}$ to $50 \mathrm{~nm}$, due to the higher surface area for ionic interactions provided by nanoapatite fillers. A molecular model is developed for the viscoelastic behavior of filled hydrogels which accounts for the effect of polymer/filler interaction energy. The model is used to predict the viscoelastic response of PLEOF/HA hydrogel nanocomposite.

\section{THEORETICAL MODEL}

The model is based on the theory of reversible adsorption from a dilute polymer solution $[15,22]$. Adsorption of the polymer chains from solution on the solid surface takes place when the chains energetically prefer the surface over the solvent. The average residence time of each monomer on the solid surface is determined by the binding energy between the monomer and particle surface. It has been shown that when the contact energy per monomer is less than the thermal energy, $k_{B} T$, the adsorption process is reversible, that is, the adsorbed polymer chain detaches from the surface after a finite residence time and the bonding site are replaced with another polymer chain [22]. When the binding energy is somewhat larger than $k_{B} T$, the adsorption becomes irreversible, and the adsorbed chains flatten and freeze on the interactive surface [23].

\subsection{Filler-gel interfacial structure}

The equilibrium configuration of a chain segment (between two consecutive cross-link points), near the filler surface with radius, $R_{f}$, is schematically shown in Figure 5 . The segment can reversibly adsorb on the colloidal surface and form a polydisperse succession of loops, tails, and sequences of bound monomers (trains). Each segment with

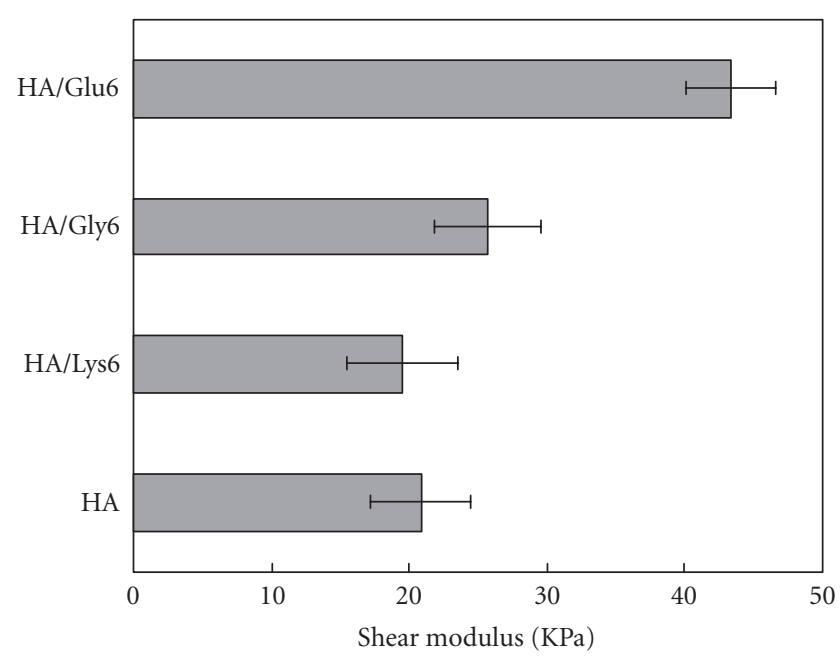

Figure 4: Comparison of the shear modulus of PLEOF/HA composites with 9 vol\% untreated nanoparticles with nanoparticles treated with Ac-Glu6, Ac-Gly6, and Ac-Lys6.

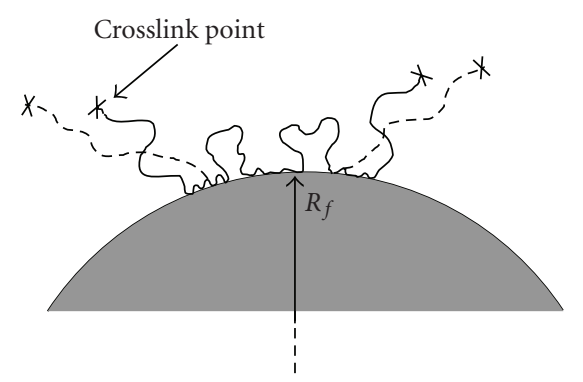

FIGURE 5: Schematic diagram of the equilibrium configuration of an adsorbed polymer segment (solid line) between two crosslink points on the filler surface. The adsorbed chain consists of loops, tails, and sequences of bonded monomers. The adsorbed segment can detach from the surface at a number of points after the application of deformation (dashed line).

$N$ monomers of size $a$ occupies a spherical volume with a radius comparable with the Flory radius, $R_{F}=a N^{3 / 5}$.

In order to describe the structure of the adsorbed and fully-equilibrated polymer layer on the filler surface, we used a modified version of de Gennes scaling theory [24] for reversible adsorption from dilute solutions under good solvent conditions. The chain configuration in an adsorbed layer is determined by the competition between excluded volume, surface energy, and chain entropic effects. Assuming that the loops are extended to an average thickness $D$ from the surface, the fraction of monomers in direct contact with the particle surface can be approximated by $f \cong a / D$. Assuming that the conformational entropy and energetic affinity with the surface are the only factors that determine the configuration of the adsorbed layer, the free energy per segment, $\Psi$, can be written as [25]

$$
\Psi \cong k_{B} T\left(\frac{R_{F}}{D}\right)^{5 / 3}-f N \Delta E_{\mathrm{ad}}
$$


Minimizing the free energy with respect to $D$ yields

$$
f \cong\left(\frac{\Delta E_{\mathrm{ad}}}{k_{B} T}\right)^{3 / 2}
$$

\subsection{Dynamics of the adsorbed layer}

The gel-particle energetic attraction is modeled as a frictional interaction between the adsorbed monomers and particle surface, in addition to the regular monomer-solvent and/or monomer-monomer frictions. Therefore, the total friction coefficient due to the hydrodynamic force acting on the $i$ th monomer is [26]

$$
\begin{aligned}
& (\xi)_{i}=\xi_{1}, \quad \text { ith monomer is adsorbed, } \\
& (\xi)_{i}=\xi_{0}, \quad \text { ith monomer is not adsorbed, }
\end{aligned}
$$

where $\xi_{1}$ is the friction coefficient due to monomer-particle interaction and $\xi_{0}$ is the friction coefficient corresponding to the self-diffusion of a single monomer and it accounts for its friction with the solvent molecules and/or other nonadsorbed monomers. Using an Arrhenius-type activation model for a monomer of size $a$, the friction coefficient is approximated by $\xi_{0} \cong k_{B} T \tau_{0} / a^{2}$, with time constant $\tau_{0}$ defined by

$$
\tau_{0}=\tau^{*} \exp \left(\frac{E_{0}}{k_{B} T}\right)
$$

where $\tau^{*}$ is a constant. A similar activation model can be used to estimate $\xi_{1} \cong k_{B} T \tau_{1} / a^{2}$, where $\tau_{1}$ is defined as

$$
\tau_{1}=\tau^{*} \exp \left(\frac{E_{1}}{k_{B} T}\right) .
$$

Here, $E_{1}=\Delta E_{\mathrm{ad}}+E_{0}$ is the energy required to detach the adsorbed monomer from the particle surface.

Since a fraction $f$ of the monomers in an adsorbed segment is in contact with the particle surface, the total friction coefficient of the entire adsorbed segment is given by

$$
\xi_{a}=N\left(f \xi_{1}+(1-f) \xi_{0}\right),
$$

where by using (4) and (5),

$$
\xi_{1}=\xi_{0} \exp \frac{\Delta E_{\mathrm{ad}}}{k_{B} T} .
$$

For weakly attractive surfaces, segments are partially adsorbed to the surface and exhibit their 3D Rouse dynamics [27]. Hence, the relaxation time of the adsorbed segment is

$$
\tau_{a} \cong R_{F}^{2} \frac{\xi_{a}}{k_{B} T}=\tau_{f}\left(f \exp \frac{\Delta E_{\mathrm{ad}}}{k_{B} T}+(1-f)\right),
$$

where $\tau_{f} \cong R_{F}^{2}\left(\xi_{f} / k_{B} T\right)$ is the relaxation time of a free segment.

The self-similar grid structure [22] describes the adsorbed layer as a semi-dilute solution of the polymer with continuously varying local concentration of the monomers, such that at any distance $r$ from the surface, the local blob size is equal to $r$. Therefore, the equilibrium thickness of the layer is on the order of $R_{F}$. In a cross-linked system, due to the fixed-end constraint, segments cannot diffuse independently like linear chains. Hence, the adsorption-desorption process takes place between those segments which are located within the interphase region with thickness $R_{F}$ around the fillers and with total population density equal to $N_{f}^{p}+N_{a}$. Here, $N_{a}$ is the number density of the adsorbed segments and $N_{f}^{p}$ represents the number density of free segments within the interphase zone, which are able to participate in the adsorption-desorption process. The rate of attachment can be shown by the following kinetic equation:

$$
\frac{d N_{a}}{d t}=N_{f}^{p}\left(\tau_{\text {ads }}\right)^{-1}-N_{a}\left(\tau_{\text {des }}\right)^{-1},
$$

where $\tau_{\mathrm{ads}}$ and $\tau_{\text {des }}$ are the characteristic times of adsorption and desorption of the segments, respectively.

The energy required for detachment of an adsorbed segment is equal to $f N \Delta E_{\mathrm{ad}}$. In the presence of an applied macrodeformation, the tails of each segment move with the bulk material (Figure 5). The detachment process is thus favored by the resultant entropic tension exerted by the segment. Considering this effect, the time constants associated with the attachment and detachment of the segments follow the relation defined by

$$
\tau_{\text {des }}=\tau_{\text {ads }} \exp \left[\frac{f N \Delta E_{\mathrm{ad}}-\delta F_{a}}{k_{B} T}\right],
$$

where $F_{a}$ is the entropic force in the segment and $\delta$ is an activation length on the order of the displacement required to detach the bound segment from the particle surface. The desorption of a bound monomer with weak and short range interaction with the adsorbing surface can be considered as a local process. It takes place when the monomer diffuses a distance on the order of the equilibrium size of the first blob in contact with the wall [28]. According to the selfsimilar grid structure theory [22], the size of the first blob in contact with the particle surface is on the order of the size of a monomer. Therefore, $\delta$, the total displacement required to separate the entire segment with $f$ fraction of adsorbed monomers, is $a \leq \delta \leq R_{F}$.

\subsection{Macroscopic properties}

The classical Maxwell model [29] is used to describe the viscoelasticity of the matrix. It is assumed that the deformations are relatively small such that geometric nonlinearities can be neglected and only the thixotropic nonlinearities, due to polymer-filler interactions, are considered. At any instant in time, a representative segment is either adsorbed to the surface of the particle or it is free. Assuming that the configurations of free and adsorbed segments evolve independently, the total stress in the composite is therefore the sum of the stresses by the adsorbed $\left(\boldsymbol{\sigma}_{a}\right)$ and free $\left(\boldsymbol{\sigma}_{f}\right)$ segments, that is,

$$
\boldsymbol{\sigma}=\boldsymbol{\sigma}_{a}+\boldsymbol{\sigma}_{f} .
$$


The contribution of the segments to the stress tensor is given by Kramers expression:

$$
\begin{gathered}
\boldsymbol{\sigma}_{a}=3 G_{a} \frac{\left\langle\mathbf{R}_{a} \mathbf{R}_{\boldsymbol{a}}\right\rangle}{R_{F}^{2}}, \\
\boldsymbol{\sigma}_{f}=3 G_{f} \frac{\left\langle\mathbf{R}_{f} \mathbf{R}_{f}\right\rangle}{R_{F}^{2}},
\end{gathered}
$$

where $G_{a}$ and $G_{f}$ represent the stiffness of the adsorbed segments and free segments (located out of the interphase zone), respectively. $\mathbf{R}_{i}(i=a, f)$ is the segment end-to-end vector and $\langle\cdots\rangle$ shows the ensemble average. According to the classical theory of rubber elasticity [30], a linear dependency is introduced between the modulus and number density of the chains at constant temperature, that is, $G_{i} \propto$ $N_{i}(i=a, f)$. Therefore, at steady-state conditions, we have

$$
G_{a}=G_{f}^{p} \exp \left[\frac{f N \Delta E_{\mathrm{ad}}-\delta F_{a}}{k_{B} T}\right],
$$

where $G_{f}^{p}$ shows the stiffness of the free segments within the interphase zone.

The mechanical response of the network can be decoupled into two parts: a rate independent response and a time dependent deviation from the equilibrium [31], that is,

$$
\boldsymbol{\sigma}_{i}=\boldsymbol{\sigma}_{i}^{e}+\boldsymbol{\sigma}_{i}^{v}, \quad i=a, f,
$$

where $\boldsymbol{\sigma}_{i}^{e}$ and $\boldsymbol{\sigma}_{i}^{v}$ stand for the rate independent and rate dependent components of the stress, respectively. Using (12), the time independent component of stress can be expressed by

$$
\boldsymbol{\sigma}_{i}^{e}=G_{i} \mathbf{F} \cdot \mathbf{F}^{T}, \quad i=a, f,
$$

where $\mathbf{F}$ is the deformation gradient tensor.

In their simplest form, the constitutive relations for the evolution of the rate dependent stresses produced by the segments can be expressed by the Maxwell (upperconvected) equations [29]:

$$
\begin{aligned}
\tau_{a} \hat{\boldsymbol{\sigma}}_{a}^{v}+\boldsymbol{\sigma}_{a}^{v}-G_{a} \mathbf{I} & =0, \\
\tau_{f} \hat{\boldsymbol{\sigma}}_{f}^{v}+\boldsymbol{\sigma}_{f}^{v}-G_{f} \mathbf{I} & =0,
\end{aligned}
$$

where $\mathbf{I}$ is the identity tensor. Here, $\hat{\boldsymbol{\sigma}}$ designates the upperconvected derivative of the stress tensor given by $\hat{\boldsymbol{\sigma}}=\partial \boldsymbol{\sigma} / \partial t-$ $\boldsymbol{\sigma} \cdot \mathbf{L}_{\mathrm{ef}}-\mathbf{L}_{\mathrm{ef}}^{\mathrm{T}} \cdot \boldsymbol{\sigma}$, where $\mathbf{L}_{\mathrm{ef}}=h(\phi) \nabla \mathbf{v}$ is the effective velocity gradient tensor and $\mathbf{v}$ is the velocity field. Here, $h(\phi)$ accounts for the hydrodynamic interaction between the particles with volume fraction $\phi$. This is based on a phenomenological consideration that the effective velocity gradient experienced by the polymer matrix is higher than the externally-applied velocity gradient, due to the rigidity of the filler particles. The contribution of the hydrodynamic effect is determined by the shape and volume fraction of the particles [32]. At low filler concentrations, it is represented by

$$
h(\phi)=1+\zeta \phi
$$

where the prefactor parameter $\zeta$ accounts for the particle geometry.

\subsection{Model predictions}

The model is used to predict the effect of HA surface adsorption energy on the overall steady-state shear modulus of the PLEOF hydrogel composites. Assuming the material is under oscillatory shear strain with frequency $\omega$, the dynamic strain can be stated as

$$
\lambda(t)=\lambda_{0} \sin \omega t
$$

For simplicity, only the affine (time independent) part of the deformation is considered for evaluation of the entropic force in (13). For oscillatory shear loading with small strain $\lambda_{0}$, the deformation gradient can be written as

$$
\mathbf{F}=\left(\begin{array}{ccc}
1 & \lambda_{0} \sin \omega t & 0 \\
0 & 1 & 0 \\
0 & 0 & 1
\end{array}\right)
$$

The average end-to-end vector of an adsorbed segment during a period of oscillation can be obtained by

$$
\overline{\mathbf{R}}_{a}=\overline{\mathbf{F}} \cdot \mathbf{R}_{F},
$$

where the components of $\overline{\mathbf{F}}$ are the average of the absolute values of the corresponding components in $\mathbf{F}$ over one period of oscillation. The mean square end-to-end distance, given by (20), was used in $F_{a}=\left(3 k_{B} T / R_{F}^{2}\right) \bar{R}_{a}\left(1-\bar{R}_{a}^{2} / R_{a, \text { max }}^{2}\right)^{-1}$ the Warner approximation for the entropic force, to calculate the average entropic tension in an adsorbed segment.

$R_{a, \max } / R_{F}, c=\Delta E_{\mathrm{ad}} / k_{B} T$, and $\delta / R_{F}$ are the model parameters which represent the characteristic length of the polymer segments near the particle surface and the interaction energy between the PLEOF segments and HA nanoparticles. These parameters are independent of the filler concentration. $G_{f}^{p} / G_{f}$ is another fitting parameter which is proportional to the volume fraction of interphase zone and number of those free chains, located in the interphase zone, contributing to the adsorption-desorption kinetics. Therefore, this parameter changes with the size and concentration of nanoparticles. The magnitude of the shear modulus in the low strain region is found to be sensitive to the values of $c$, while the onset of nonlinearity in the viscoelastic response is controlled by $\delta$ and $R_{a, \max }$.

The Flory radius of the segments between two consecutive fumarate units in the PLEOF (i.e., potential crosslink points) is estimated to be approximately $10 \mathrm{~nm}$. The hydrodynamic factor $\zeta$ is set equal to 2.5 considering the spherical shape of HA nanoparticles. The best fit of the experimental results to the model was obtained with $G_{f} \cong$ $4 \mathrm{kPa}$ and $\tau_{f}=0.001$ second for the free segments. Other extracted fitting parameters are li sted in Table 1. The value of $c$ for the surface-treated samples was found to be higher than that of untreated samples, due to the stronger average monomer-filler interaction in the presence of AcGlu6 peptide.

Figure 3 also represents the predicted values of small strain shear modulus of the Ac-Glu6 treated nanocomposites at different volume fraction of nanoapatite particles. Fitting the model parameters with measured storage modulus of 


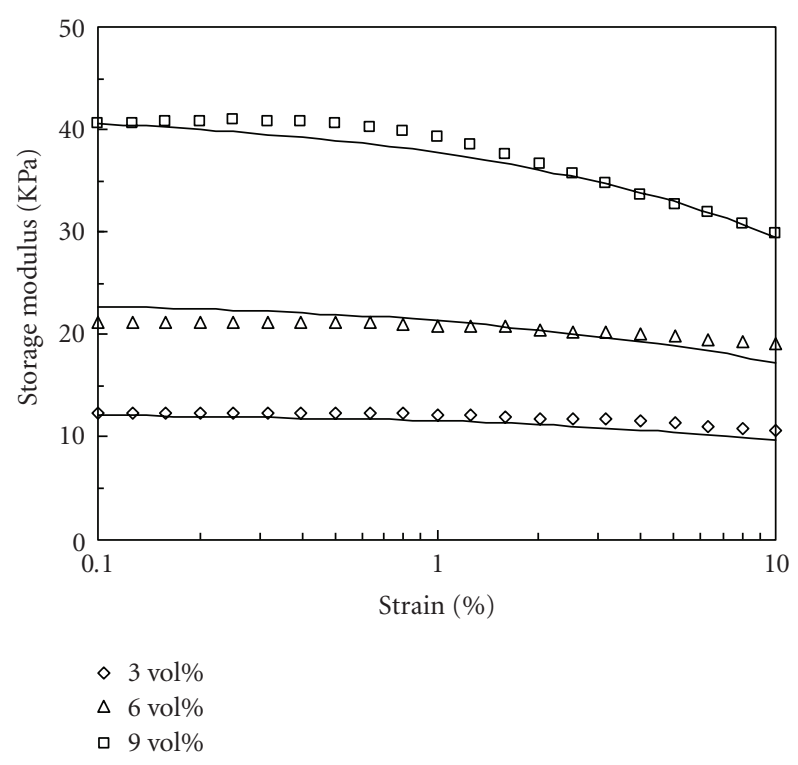

(a)

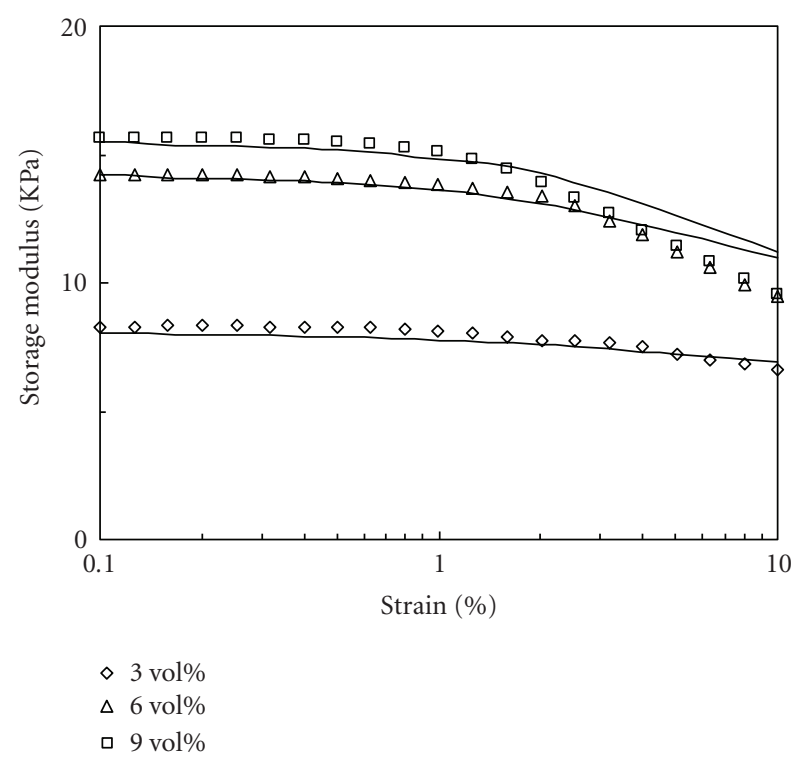

(b)

FIGURE 6: Comparison of the experimental results with model predictions (solid lines) for the storage modulus of PLEOF/HA composites, prepared with 3-9 vol\% of (a) treated and (b) untreated nanoparticles as a function of strain amplitude (frequency $=1 \mathrm{~Hz}$ ).

TABLE 1: Fitted parameters of the proposed model for the hydrogel/nanoapatite composites.

\begin{tabular}{|c|c|c|}
\hline \multicolumn{3}{|c|}{ Parameters independent from filler concentration } \\
\hline$\delta / R_{F}=0.5, R_{a, \max } / R_{F}=1.5$ & $\begin{array}{c}\text { Treated } \\
c=0.02\end{array}$ & $\begin{array}{l}\text { Untreated } \\
c=0.012\end{array}$ \\
\hline \multicolumn{3}{|c|}{ Parameters variable with filler concentration } \\
\hline$\phi$ & $\begin{array}{l}\text { Treated } \\
G_{f}^{p} / G_{f}\end{array}$ & $\begin{array}{c}\text { Untreated } \\
G_{f}^{p} / G_{f}\end{array}$ \\
\hline $3(\operatorname{vol} \%)$ & 0.25 & 0.33 \\
\hline $6(\operatorname{vol} \%)$ & 0.53 & 0.75 \\
\hline $9(\operatorname{vol} \%)$ & 0.93 & 0.76 \\
\hline
\end{tabular}

microapatite composites results in a negligible value for $G_{f}^{p} / G_{f}$, and consequently the model prediction is fairly close to $h(\phi) G_{f}$, that is, the Guth-Smallwood equation. Hence, for large HA particles, relative to the length of the interacting segment, the reinforcement is dominated by hydrodynamic effects. It should be mentioned that due to constant thickness of interphase zone $\left(\sim R_{F}\right)$, reduction of the filler size increases the volume fraction of the interphase zone in the matrix. As a result, the values of $G_{a}$ and $G_{f}^{p}$ and consequently the overall shear modulus of the composite increase.

The model results and experimental values for the shear storage modulus of surface treated and untreated composites are shown in Figure 6 as a function of strain amplitude. The model predictions qualitatively follow the trends in the experimentally-measured values; however, there are discrepancies, especially between the model results and experimental data of untreated samples.
The higher adsorption energy between PLEOF segments and HA nanoparticles in Glu6-treated samples (indicated by parameter $c=\Delta E_{\mathrm{ad}} / k_{B} T$ in Table 1) can be attributed to the strong ionic bond with high adsorption energy between the negatively-charged glutamic acid sequences and the calcium ions on the surface of HA particles, compared to the weaker polar interactions in the absence of Ac-Glu6. The exponential dependence of $G_{a}$ on adsorption energy, as shown by (13), implies that increasing the polymer-surface interaction energy leads to a significant enhancement in linear viscoelastic properties of the polymer composites. On the other hand, lower modulus of the untreated samples indicates weaker polymer-filler interfacial bonds in those samples which may give rise to stronger tendency for HA particles to aggregate, as a result of interparticle electrostatic or van der Waals' interactions [33]. The network of locally aggregated particles follows a different kinetic and relaxation pattern rooted in the stored elastic energy in the strained clusters and the failure properties of filler-filler bonds [34]. This mechanism, which is not accounted in the proposed model, can be considered as the major source of discrepancy between the experimental data of untreated samples and the model predictions.

\section{CONCLUSIONS}

The viscoelastic properties of a multifunctional bone mimetic nanocomposite with a matrix-apatite adhesion mechanism similar to that of the natural bone were investigated. A glutamic acid-rich peptide (a sequence of 6 glutamic acids), derived from osteonectin, was functionalized with an acrylate group (Ac-Glu) for covalent attachment to the matrix. The biodegradable in situ cross-linkable poly 
(lactide-co-ethylene oxide fumarate) (PLEOF) hydrogel and HA crystals were used to mimic the gelatinous matrix and mineral phases of the bone, respectively. HA particles were treated with Ac-Glu6 and dispersed in the aqueous PLEOF mixture. The polymerizing mixture was cross-linked with a neutral redox initiation system and the gelation process was monitored in situ as a function of time with a rheometer. The rheometry results showed that the Ac-Glu6 peptide significantly enhanced the viscoelastic properties of the hydrogel/HA nanocomposite. A molecular model was developed to predict the role of polymer-apatite interaction in improving the viscoelastic behavior of the synthesized bone mimetic hydrogel nanocomposite. The scaling law of de Gennes for equilibrium reversible polymer adsorption in good solvent conditions was used to predict the equilibrium configuration of the adsorbed polymer layer on the surface of HA particles. The relaxation and diffusion of the adsorbed segments were predicted using a Maxwell type kinetic model. Predictions of the model are compared with the measured dynamic shear modulus of the PLEOF hydrogel reinforced with HA nanoparticles. The measured rheological and viscoelastic characteristics and the predictions of the model can be used to control injectability, in the in situ hardening phase, and to predict mechanical properties in the postgelation phase for composite scaffolds used in skeletal tissue regeneration.

\section{ACKNOWLEDGMENT}

This work was supported by grants from the Arbeitsgemeinschaft Fur Osteosynthesefragen (AO) Foundation (AORF Project 05-J95) and the Aircast Foundation.

\section{REFERENCES}

[1] N. A. Peppas and R. Langer, "New challenges in biomaterials," Science, vol. 263, no. 5154, pp. 1715-1720, 1994.

[2] D. C. Tancred, B. A. O. McCormack, and A. J. Carr, "A synthetic bone implant macroscopically identical to cancellous bone," Biomaterials, vol. 19, no. 24, pp. 2303-2311, 1998.

[3] L. Hangody, G. Kish, Z. Kárpáti, I. Udvarhelyi, I. Szigeti, and M. Bély, "Mosaicplasty for the treatment of articular cartilage defects: application in clinical practice," Orthopedics, vol. 21, no. 7, pp. 751-756, 1998.

[4] J. S. Temenoff and A. G. Mikos, "Injectable biodegradable materials for orthopaedic tissue engineering," Biomaterials, vol. 21, no. 23, pp. 2405-2412, 2000.

[5] S. S. Liao, F. Z. Cui, W. Zhang, and Q. L. Feng, "Hierarchically biomimetic bone scaffold materials: nano-HA/collagen/PLA composite," Journal of Biomedical Materials Research. Part B, vol. 69, no. 2, pp. 158-165, 2004.

[6] K. E. Tanner, R. N. Downes, and W. Bonfield, "Clinical applications of hydroxyapatite reinforced materials," British Ceramic Transactions, vol. 93, no. 3, pp. 104-107, 1994.

[7] A. L. Boskey, "Will biomimetics provide new answers for old problems of calcified tissues?" Calcified Tissue International, vol. 63, no. 3, pp. 179-182, 1998.

[8] J. E. Zerwekh, S. Kourosh, R. Scheinberg, et al., "Fibrillar collagen-biphasic calcium phosphate composite as a bone graft substitute for spinal fusion," Journal of Orthopaedic Research, vol. 10, no. 4, pp. 562-572, 1992.
[9] J. A. Buckwalter, M. J. Glimcher, R. R. Cooper, and R. Recker, "Bone biology. Part II: formation form, modeling, remodeling, and regulation of cell function," Journal of Bone and Joint Surgery, vol. 77, no. 8, pp. 1276-1289, 1995.

[10] V. I. Sikavitsas, J. S. Temenoff, and A. G. Mikos, "Biomaterials and bone mechanotransduction," Biomaterials, vol. 22, no. 19, pp. 2581-2593, 2001.

[11] M. F. Young, "Bone matrix proteins: their function, regulation, and relationship to osteoporosis," Osteoporosis International, vol. 14, supplement 3, pp. S35-S42, 2003.

[12] R. Fujisawa, Y. Wada, Y. Nodasaka, and Y. Kuboki, "Acidic amino acid-rich sequences as binding sites of osteonectin to hydroxyapatite crystals," Biochimica et Biophysica Acta, vol. 1292, no. 1, pp. 53-60, 1996.

[13] R.-L. Xie and G. L. Long, "Elements within the first 17 amino acids of human osteonectin are responsible for binding to type V collagen," Journal of Biological Chemistry, vol. 271, no. 14, pp. 8121-8125, 1996.

[14] X. He and E. Jabbari, "Solid-phase synthesis of reactive peptide crosslinker by selective deprotection," Protein and Peptide Letters, vol. 13, no. 7, pp. 715-718, 2006.

[15] P. G. de Gennes, "Polymers at an interface: a simplified view," Advances in Colloid and Interface Science, vol. 27, no. 3-4, pp. 189-209, 1987.

[16] A. S. Sarvestani, X. He, and E. Jabbari, "Viscoelastic characterization and modeling of gelation kinetics of injectable in situ cross-linkable poly(lactide-co-ethylene oxide-co-fumarate) hydrogels," Biomacromolecules, vol. 8, no. 2, pp. 406415, 2007.

[17] E. Guth, "Theory of filler reinforcement," Journal of Applied Physics, vol. 16, no. 1, pp. 20-25, 1945.

[18] N. H. de Leeuw, "A computer modelling study of the uptake and segregation of fluoride ions at the hydrated hydroxyapatite (0001) surface: introducing a $\mathrm{Ca}_{10}\left(\mathrm{PO}_{4}\right)_{6}(\mathrm{OH})_{2}$ potential model," Physical Chemistry Chemical Physics, vol. 6, no. 8, pp. 1860-1866, 2004

[19] W. R. Walsh and N. Guzelsu, "Compressive properties of cortical bone: mineral-organic interfacial bonding," Biomaterials, vol. 15, no. 2, pp. 137-145, 1994.

[20] H. W. Choi, H. J. Lee, K. J. Kim, H.-M. Kim, and S. C. Lee, "Surface modification of hydroxyapatite nanocrystals by grafting polymers containing phosphonic acid groups," Journal of Colloid and Interface Science, vol. 304, no. 1, pp. 277-281, 2006.

[21] E. I. F. Pearce, "Ion displacement following the adsorption of anionic macromolecules on hydroxyapatite," Calcified Tissue International, vol. 33, no. 4, pp. 395-402, 1981.

[22] P. G. de Gennes, "Polymer solutions near an interface. 1. Adsorption and depletion layers," Macromolecules, vol. 14, no. 6, pp. 1637-1644, 1981.

[23] B. O'Shaughnessy and D. Vavylonis, "Non-equilibrium in adsorbed polymer layers," Journal of Physics: Condensed Matter, vol. 17, no. 2, pp. R63-R99, 2005.

[24] P.G. de Gennes, Scaling Concepts in Polymer Physics, Cornell University Press, Ithaca, NY, USA, 1985.

[25] J. Gong and Y. Osada, "Gel friction: a model based on surface repulsion and adsorption," Journal of Chemical Physics, vol. 109, no. 18, pp. 8062-8068, 1998.

[26] A. S. Sarvestani and C. R. Picu, "A frictional molecular model for the viscoelasticity of entangled polymer nanocomposites," Rheologica Acta, vol. 45, no. 2, pp. 132-141, 2005.

[27] A. L. Ponomarev, T. D. Sewell, and C. J. Durning, "Surface diffusion and relaxation of partially adsorbed polymers," Journal of Polymer Science, vol. 38, no. 9, pp. 1146-1154, 2000. 
[28] J. Wittmer, A. Johner, J. F. Joanny, and K. Binder, "Chain desorption from a semidilute polymer brush: a Monte Carlo simulation," The Journal of Chemical Physics, vol. 101, no. 5, pp. 4379-4390, 1994.

[29] G. Larson, Constitutive Equations for Polymer Melts and Solutions, Butterworths, Boston, Mass, USA, 1988.

[30] J. D Ferry, Viscoelastic Properties of Polymers, John Wiley \& Sons, New York, NY, USA, 1980.

[31] J. S. Bergström and M. C. Boyce, "Large strain time-dependent behavior of filled elastomers," Mechanics of Materials, vol. 32, no. 11, pp. 627-644, 2000.

[32] G. Huber and T. A. Vilgis, "On the mechanism of hydrodynamic reinforcement in elastic composites," Macromolecules, vol. 35, no. 24, pp. 9204-9210, 2002.

[33] A. S. Sarvestani and E. Jabbari, "Modeling and experimental investigation of rheological properties of injectable poly (lactide ethylene oxide fumarate)/hydroxyapatite nanocomposites," Biomacromoelcules, vol. 7, no. 5, pp. 1573-1580, 2006.

[34] G. Heinrich and M. Klüppel, "Recent advances in the theory of filler networking in elastomers," Advances in Polymer Science, vol. 160, pp. 1-44, 2002. 

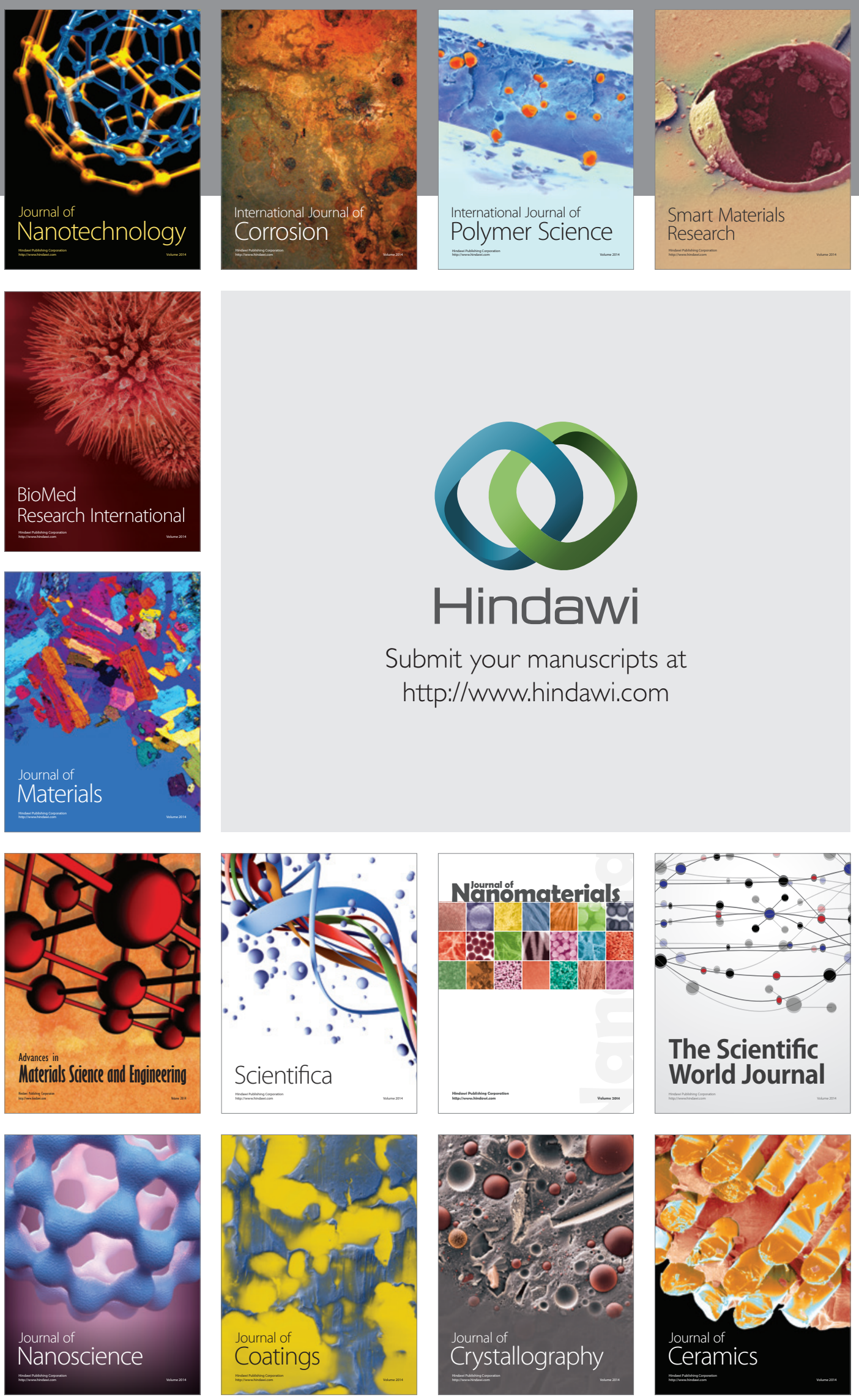

The Scientific World Journal

Submit your manuscripts at

http://www.hindawi.com

\section{World Journal}

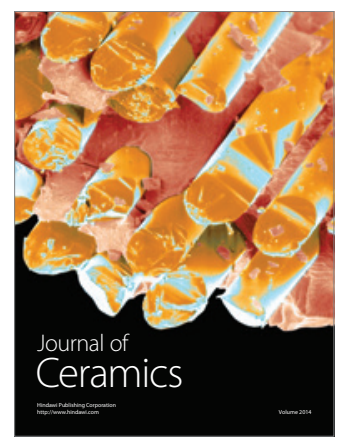

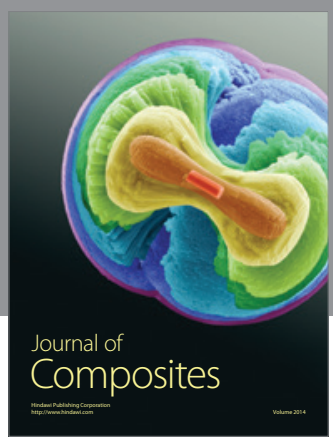
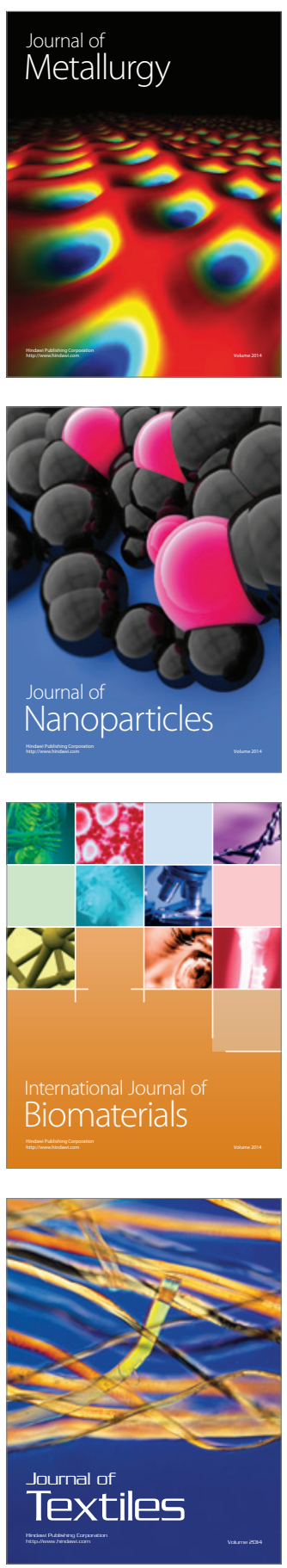